$532.595: 621.643 \cdot 752$

\title{
Comparisons between Experimental and Theoretical \\ Results of the Waterhammer with Water \\ Column Separations*
}

\author{
By Takahiko TANAHASHI** and Eiji KasaharA***
}

\begin{abstract}
The laboratory equipment used in association with the pumping plant of the cooling-water supply for steam electric stations was made in order to give validity to the analysis of the previaus paper. In this experiment, water column separations were caused by the negative pressure surge at two positions, just after the valve and at the middle of the conduit. In this paper are shown the effects of : the time of gate closure : normal head of the pump : and normal velocity on the first or the second pressure rise due to the rejoining, and the relationship between the time of the maximum pressure rise and the normal velocity. The theoretical results on the assumption that the boundary between the vapor void and the water column is a free surface agreed well with the test results over the range in which the second pressure rise is caused.
\end{abstract}

\section{Introduction}

A pipe system as a unit has been designed for arbitrary maximum pressure without backflow and without separation of the water column. But recently the pumping plants associated with the cooling-water supply for thermal power stations have not been able to prevent water in a conduit from separating into two parts because of the enlarged, high condensers. This subject seems to have aroused a new interest, because of the high pressure created by the rejoining of two perfectly separated water columns. This phenomenon is known to hydraulic engineers as a waterhammer with water column separation. Some works with respect to this problem have been published in recent years: Parmakian (1) (2), Bergeron ${ }^{(3)}$ and Angus( ${ }^{(4)}$ developed more fully the classical method of graphical solution without the effect of friction. Richards ${ }^{(5)}$ and Duc ${ }^{(6)}$ showed the experimental results of typical water-pumping plants. Kephart and Davis ${ }^{(i)}$ obtained the equation of estimation for maximum pressure rise in a discharge line which is equipped with check valves at the pump outlet, using the rigid water column theory. $\mathrm{Li}^{(8)}(9)$ tried to make clear the mechanisms of water column separation. Cartens and Hagler (10) compared the experimental results with classical, graphical solutions. Baltzer(11) combined the characteristic

* Received IIth August, 1969

** Assistant, Faculty of Engineering, Keio University, Koganei.

*** Professor, Faculty of Engineering, Keio University. methods of Streeter (12) and the theory of mechanism of water column separation of $\mathrm{Li}$, and discussed the differences between calculated results and experimental ones.

In a previous paper ${ }^{(13)}$ theoretical research was made on the waterhammer with water column separation. In this paper are shown the effects of : the time of gate closure; normal head of the pump; and normal velocity on the first or second pressure rise due to the rejoining, and the relationship between the time of the maximum pressure rise and the normal velocity. The theoretical results based on the previous paper agreed well with the test results over the range in which the second pressure rise is caused.

\section{Notations}

$D:$ inside diameter of conduit

$E$ : modulus of elasticity of pipe wall material

$H$ : total pressure head

$K$ : bulk modulus of water

$L:$ total length of conduit

$M^{*}:$ Mach number $=V / a$

$P$ : pressure in conduit

$R$ : inside radius of conduit

$T_{1}$ : time from valve closure to the first maximum pressure

$V:$ velocity in conduit

$Z$ : height of conduit from water leve]

$a:$ velocity of pressure wave $=\sqrt{\frac{k g}{\gamma} /\left(1+\frac{k}{E} \lambda\right)}$ 
$f:$ Darcy-Weisbach friction coefficient

$g:$ gravity acceleration

$h:$ dimensionless pressure head $=H / H_{0}$

$t:$ time

$v:$ dimensionless velocity $=V / V_{N}$

$x$ : positive distance measured from pump to reservoir

$\gamma:$ specific weight of water

$\varepsilon_{1}:$ circumferential strain $=\Delta R / R$

$\varepsilon_{2}$ : longitudinal strain $=\Delta(\delta x) / \delta x$

$\zeta^{*}$ : coefficient of hydraulic loss head $=\left(f L V_{N}^{2}\right) /\left(2 g D H_{N}\right)$

$\theta$ : angle between inclined conduit and horizontal line

$\theta^{*}:$ characteristic inclination value $=\left(V_{N} L \sin \theta\right) /\left(a H_{N}\right)$

$\lambda$ : constant determined by support conditions of conduit $=\left(2 \varepsilon_{1}+\varepsilon_{2}\right) / \Delta P$

$\xi:$ dimensionless distance $=x / L$

$\rho^{*}:$ conduit constant $=\left(a V_{N}\right) /\left(g H_{N}\right)$

$\tau:$ dimensionless time $=(a t) / L$

Subscripts

$N$ : normal value

* : dimensionless characteristic value

$i$ : position

$j:$ order in same position

$k$ : time

$a:$ atmosphere

$v$ : vapour

$R$ : reservoir

$\max 1$ : first pressure rise

$\max 2$ : second pressure rise

\section{Analysis}

3.1 Fundamental equations of elastic theory

Fundamental equations for the analysis of waterhammer are formularized ${ }^{(14)}$ by one-dimensional kinematic equation

$$
\frac{\partial V}{\partial t}+V \frac{\partial V}{\partial x}=-g\left(\frac{\partial H}{\partial x}+\frac{f}{D} \frac{V|V|}{2 g}\right)
$$

and a modified equation of continuity

$$
\frac{\partial H}{\partial t}+V\left(\frac{\partial H}{\partial x}-\sin \theta\right)=-\frac{a^{2}}{g} \frac{\partial V}{\partial x} \cdots
$$

where

$$
\begin{aligned}
& a=\sqrt{(K g / \gamma) /\left(1+\frac{K}{E}-\lambda\right)} \\
& H=(P / \gamma)+Z \\
& \lambda=\frac{E}{\Delta P}\left(2 \varepsilon_{1}+\varepsilon_{2}\right), \quad \varepsilon_{1}=\frac{\Delta R}{R}, \quad \varepsilon_{2}=\frac{\Delta \delta x}{\delta x}
\end{aligned}
$$

\subsection{Method of characteristics}

Equations ( 1 ) and (2) belong to a system of pseudolinear, hyperbolic equations. Accordingly, there exist characteristics in the $x-t$ plane and $V-H$ plane (hodograph plane) and they are expressed as follows :

$$
\begin{aligned}
& \text { For } \quad d x=(V+a) d t \\
& d H=-\frac{a}{g} d V-\left(a \frac{f}{D} \frac{|V|}{2 g}-\sin \theta\right) V d t .
\end{aligned}
$$

and

For $d x=(V-a) d t$

$$
d H=\frac{a}{g} d V+\left(a \frac{f}{D} \frac{|V|}{2 g}+\sin \theta\right) V d t
$$

When these are reduced to dimensionless equations,

For $d \xi=\left(M^{*}+1\right) d \tau$

$d h=-\rho^{*} d v-\left(\zeta^{*}|v|-\theta^{*}\right) v d \tau$

and

For $d \xi=\left(M^{*}-1\right) d \tau$

$d h=\rho^{*} d v+\left(\zeta^{*}|v|+\theta^{*}\right) v d \tau$

are obtained.

\subsection{Calculation method}

In the problem of waterhammer, $M^{*}$ is much less than unity. Therefore, $M^{*}$ is dispensed with and the characteristic curve on the $\xi-\tau$ plane is linearized to obtain $d \xi= \pm d \tau$. When $\mathrm{Eq} .(5)$ is integrated from $i-1$ to $i$, and Eq. (6) from $i+1$ to $i$ by means of the trapezoidal formula in accordance with the specific time interval method, the linear approximation to Eqs. (5) and (6) is

$$
\begin{aligned}
& \left\{h_{i, 2}{ }^{k+1}+\frac{1}{2}\left(\zeta_{i}{ }^{*}\left|v_{i, 2}{ }^{k+1}\right|-\theta_{i}{ }^{*}\right) v_{i, 2^{k+1}} \Delta \tau\right\} \\
& -\left\{h_{i-1,3}{ }^{k}-\frac{1}{2}\left(\tilde{\zeta}_{i-1}{ }^{*}\left|v_{i-1,3}{ }^{k}\right|-\tilde{\theta}_{i-1}{ }^{*}\right) v_{i-1,3}{ }^{k} \Delta \tau\right\} \\
& =-\rho^{*}\left(v_{i, 2}{ }^{k+1}-v_{i-1,3^{k}}\right) \\
& \left\{h_{i, 3}{ }^{k+1}-\frac{1}{2}\left(\tilde{\zeta}_{i}{ }^{*}\left|v_{i, 3^{k+1}}\right|+\tilde{\theta}_{i}{ }^{*}\right) v_{i, 3^{k+1}} \Delta \tau\right\} \\
& -\left\{h_{i+1,2}{ }^{k}+\frac{1}{2}\left(\zeta_{i+1}{ }^{*}\left|v_{i+1,2}{ }^{k}\right|+\theta_{i+1}{ }^{*}\right) v_{i+1,2}{ }^{k} \Delta \tau\right\} \\
& =\rho^{*}\left(v_{1,3}{ }^{k+1}-v_{i+1,2}^{k}\right)
\end{aligned}
$$

When and if a separation of water column does not occur, then $h_{i, 2}{ }^{k}=h_{i, 3}{ }^{k}$ and $v_{i, 2}{ }^{k}=v_{i, 3}{ }^{k}$ should hold against any $i$ and $k$. Also, when a separation of water column does occur, the pressure is equal to the vapour pressure within the air bubbles, i.e.,

$$
h_{i, 2}{ }^{k}=h_{i, 3}{ }^{k}=h_{v}
$$

The volume of air bubbles generated during the time increment of $\Delta \tau$ is expressed by

$$
\begin{aligned}
& \Delta V_{i}=\int_{\tau}^{\tau+\Delta \tau} S \Delta v_{i} d \tau=\frac{S}{2}\left(\Delta v_{i}{ }^{k}+\Delta v_{i}{ }^{k+1}\right) \Delta \tau \\
& \Delta v_{i}{ }^{k}=v_{i, 3}{ }^{k}-v_{i, 2}{ }^{k}
\end{aligned}
$$

When the quantity of state at a given moment $k$ is assumed to be already known, the quantity of state at the moment of $k+1$ is obtained.

3.4 Fundamental equations of rigid theory and the evaluation of pressure rise

The movement of the water column as viewed macroscopically with reference to Fig. 1 is formularized by

$$
\begin{aligned}
& \frac{(L-X)}{g} \frac{d V}{d t}=\frac{P_{v}-P_{R}}{\rho g}-f \frac{(L-X)}{D} \frac{V|V|}{2 g} \ldots \\
& V=d X / d t \ldots \ldots \ldots \ldots \ldots \ldots \ldots \ldots \ldots \ldots \ldots \ldots \ldots \ldots \ldots \ldots \ldots \ldots \ldots \ldots \ldots \ldots \ldots \ldots \ldots \ldots \ldots \ldots
\end{aligned}
$$




$$
\frac{P_{R}}{\gamma}=H_{R}+\frac{P_{a}}{\gamma}
$$

Setting $Y=V^{2}$, linearizing of Eq. (10) by means of $\frac{d Y}{d X}=2 \frac{d V}{d t}$ and substituting Eqs. (11) and (12) into Eq. (10) give the following equation:

$$
\frac{L-X}{2 g} \frac{d Y}{d X}=-\frac{L B}{2 g} \mp f \frac{(L-X)}{D} \frac{Y}{2 g}
$$

Here the notation $\mp$ becomes minus when $V>0$ and plus when $V<0$.

$$
B=\frac{2\left(P_{a}+\gamma H_{R}-P_{v}\right)}{\rho L}=\frac{2\left(H_{a}+H_{R}-H_{v}\right) g}{L}
$$

If the quantity of separation of water column is generally small, [viz., $X \ll L$ in Eq. (13),] the solution passing through point $\left(X_{1}, Y_{1}\right)$ is given by

$$
Y=\left(Y_{1} \pm \frac{D B}{f}\right) e^{\mp(f / D)\left(x-x_{2}\right)} \mp \frac{D B}{f}
$$

Accordingly, if $V_{1}$ is equated to $\sqrt{Y_{1}}$,

$$
\begin{gathered}
V=\sqrt{\left(V_{1}{ }^{2}+\frac{D B}{f}\right) \exp \left\{-\frac{f}{D}\left(X-X_{1}\right)\right\}-\frac{D B}{f}} \\
\quad \text { for } V>0 \\
V=-\sqrt{\left(V_{1}{ }^{2}-\frac{D B}{f}\right) \exp \left\{\frac{f}{D}\left(X-X_{1}\right)\right\}+\frac{D B}{f}} \\
\text { for } V<0
\end{gathered}
$$

are obtained.

These equations indicate the movement on $V-X$ plane. Accordingly, when an initial velocity $V_{N}$ is given, the separation of water column develops up to $X_{b}$ and the flow of the fluid changes its direction in reverse and hits the valve at the final velocity of $V_{T}$. This movement is shown in Fig. 2. The conditions under which they pass through point $\left(O, V_{N}\right)$ are expressed by

$$
V=\sqrt{\left(V_{N}{ }^{2}+\frac{D B}{f}\right) \exp \left(-\frac{f X}{D}\right)-\frac{D B}{f}}
$$

Accordingly, when $V$ is equated to 0 in Eq. (18), the maximum growth distance $X_{b}$ of the separation of the water column becomes

$$
X_{b}=\frac{D}{f} \ln \left(1+\frac{f V_{N}{ }^{2}}{D B}\right)
$$

Also, since Eq. (17) passes points $\left(X_{b}, O\right)$ and (O, $V_{T}$ ), the final velocity $V_{T}$ can be obtained as

$$
\begin{aligned}
V_{T} & =-\sqrt{\frac{D B}{f}\left\{1-\exp \left(-\frac{f X_{b}}{D}\right)\right\}} \\
& =-\frac{V_{N}}{\sqrt{1+\left(f V_{N}{ }^{2} / D \bar{B}\right)}} \cdots \cdots \cdots \cdots
\end{aligned}
$$

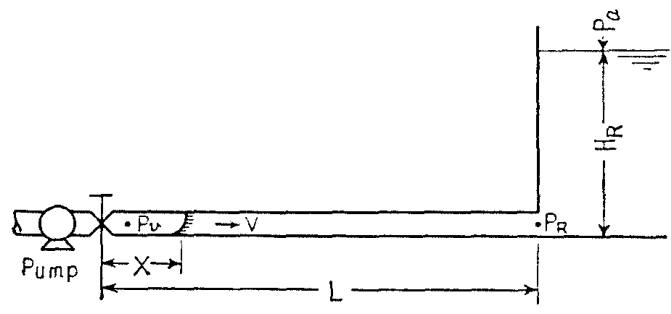

Fig. 1 Simple conduit model
Then, the maximum pressure rise is

$$
H_{\max }=\left(\frac{a V_{N}}{g}\right) / \sqrt{1+\frac{f V}{D} N^{2}}
$$

or when it is non-dimensionalized

$$
h_{\max }=\rho^{*} / \sqrt{1+\frac{\zeta^{*}}{h_{a}+h_{R}-h_{v}}} \text {. }
$$

The numerators of Eqs. (21) and (22) mean the maximum pressure rise occurring when there is no friction loss in the pipe. When there is some friction loss, Eqs. (21) and (22) indicate that the pressure rise is hampered by the value of the denominators. The above comparison between the elastic theory and the rigid theory reveals the following characteristics. While the elastic theory enables us to calculate the detailed transient phenomena in relation to the changes in time, it requires much time to calculate and is unable to grasp the general tendency of pressure rise caused by the changes in parameters, such as the water level of reservoir, normal velocity of flow, the length and form of pipe. On the other hand, the rigid theory is capable of pressure rise evaluation reflecting the changes in the various parameters relating to a simple model, and thus facilitates an estimate of the pressure rise.

3.5 Time needed to produce the maximum pressure rise

$$
\begin{aligned}
& \text { If } X \ll L \text { in Eq. (10), } \\
& d t=(2 d V) /\left(-B \mp \frac{f}{D} V^{2}\right)
\end{aligned}
$$

is obtained.

Especially, $\quad d t=-2 d V /\left(B+\frac{f}{D} V^{2}\right)$ for $V>0$

The solution of the initial condition, i.e., $V=V_{N}$ at $t=0$ is

$$
t=\frac{2}{\sqrt{\frac{B f}{D}}}\left\{\tan ^{-1} V_{N} \sqrt{\frac{f}{B D}}-\tan ^{-1} V \sqrt{\frac{f}{B D}}\right\}
$$

Here, since $V=0$ when the water column reaches the highest point, the time needed to reach that point, $T_{\text {up }}$ is

$$
T_{\mathrm{up}}=\frac{2}{\sqrt{\frac{\overline{B f}}{D}}} \tan ^{-1} V_{N} \sqrt{\frac{f}{\bar{B} \bar{D}}}
$$

Now, let us consider the case in which the flow is

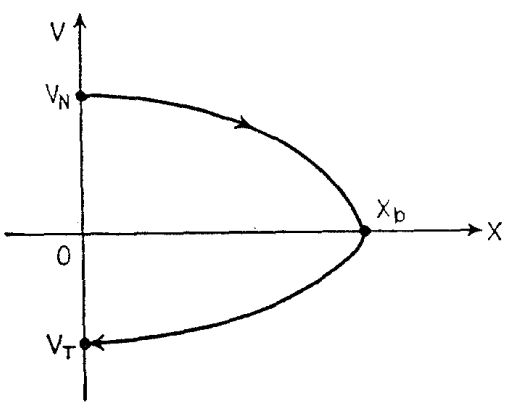

Fig. 2 Movement of water column on the $X \cdot V$ plane 
reversed. We have

$$
d t=(-2 d V) /\left(B-\frac{f}{D} V^{2}\right) \text { for } V<0
$$

Since the initial conditions are $V=0$ at $t=0$, its solution is

$$
t=\frac{1}{\sqrt{\frac{B f}{D}}} \ln \frac{\sqrt{B}-\sqrt{\frac{f}{D}} V}{\sqrt{B}+\sqrt{\frac{f}{D}} V}
$$

Accordingly, the time needed for the raised water column to hit the valve, $T_{\text {down }}$ is

$$
T_{\text {down }}=\frac{1}{\sqrt{\frac{B f}{D}}} \ln \frac{\sqrt{B}-\sqrt{\frac{f}{D}} V_{T}}{\sqrt{B}+\sqrt{\frac{f}{D}} V_{T}}
$$

The time needed for a complete cycle of the water column $T_{1}$ is $T_{1}=T_{\text {up }}+T_{\text {down }}$. When Eq. (20) is substituted into Eq. (27),

$$
\begin{gathered}
T_{1}=\sqrt{\frac{D}{f B}\left\{2 \tan ^{-1} V_{N} \sqrt{\frac{f}{B D}}\right.} \\
\left.+\ln \left(\frac{1+\frac{V_{N} \sqrt{f / B D}}{\sqrt{1+f V_{N}^{2} / B D}}}{1-\frac{V_{N} \sqrt{f} / B D}{\sqrt{1+f V V_{N}^{2} / B D}}}\right)\right\}
\end{gathered}
$$

\section{Test equipment and methods of test}

Figures 3 ( $a$ ) and ( b ) give a whole view of the test equipment and a side view extending along the coordinate axis. Figure 4 shows the acryl resin window through which the separation of the water column is observed and the reservoir section. The water pumped up from the water pool by the main pump A through a 3 -inch pipe is delivered to a narrow caliber pipe of 2 inches in diameter before it passes through the electric valve. The main pump is of the variable-stroke type, capable of changing smoothly the number of strokes from $120 \mathrm{rpm}$ to $1200 \mathrm{rpm}$. This is so designed as to change the initial velocity of the flow within a pipe. Between the delivery of the main pump and the electric valve a precision pressure guage is installed to monitor the initial pressure at the delivery. The water which passes the electric valve flows through the electromagnetic flow meter $C$ and then through the position of the installed pressure terminals 2, 3 and 4 and reaches the acryl resin window in the center of the pipe. The electric valve is operated by means of a reduction gear, and the hand'e A shifts the gear into high, medium and low speeds, while the handle $B$ gives speed combinations of $1,2,3,4$ and 5. These two handles are capable of closing the valve at 13 different speeds, except the reverse. The valve moves in axial direction at a uniform speed over $15 \mathrm{~mm}$ stroke and the distance of the valve transferred is monitored by rheostat. The valve is a round plate with a diameter of $53 \phi$. To measure the characteristics of the valve, two holes are bored on the pipe on each side of the valve. In order that the pressure terminals may not interfere with the flow of water within the pipe, they are installed in such a manner that their tips can touch the inner wall of the pipe. The pressure terminals used are pressure detectors monitoring the pressure by means of the piezo-electric effect. The pressure thus monitored is converted into voltage, which is conducted through the constant-voltage device and the bridge balanced circuit and is connected with the amplifier. Even if the valve is moved at a uniform speed, it is difficult to translate the volume of flow into a linear motion. According$l y$, in the present experiment, the change in the volume of flow over time is monitored by means of an electro-magnetic flow meter $C$. Through the acryl resin pipe

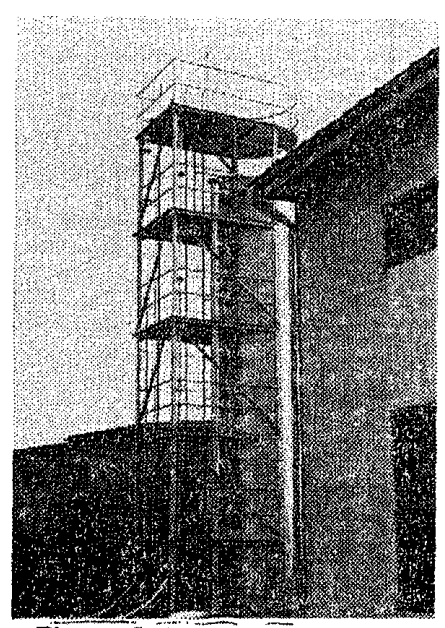

Fig. 4 Laboratory equipment (b) Pipe line
Fig. 3
Total length $120.72 \mathrm{~m}$

Each interval of conduit

(1) $\rightarrow$ (2) $13.94 \mathrm{~m} \quad$ (3) $\rightarrow$ (4) $14.08 \quad$ (5) $\rightarrow$ (5) $18.68 \mathrm{~m} \quad$ (1) $\rightarrow$ (8) $13.91 \mathrm{~m}$

(2) $\rightarrow$ (3) $14.02 \mathrm{~m} \quad$ (4) $\rightarrow$ (5) $18.36 \mathrm{~m} \quad$ (1) $\rightarrow$ (1) $13.82 \mathrm{~m} \quad$ (8) $\rightarrow$ (9) $13.91 \mathrm{~m}$ (a) Experimental apparatus

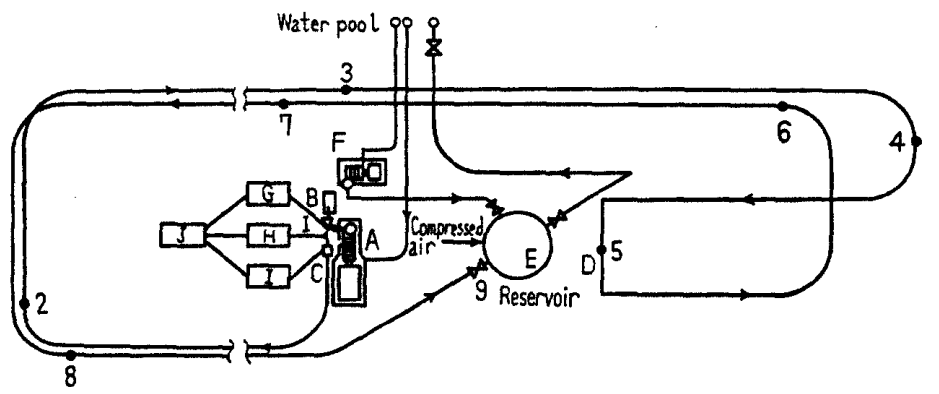

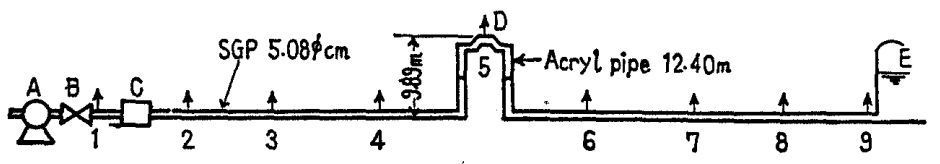


fitted into the pipe at the mid-point, pictures were aken of the processes of the separation of the water columns and their re-combination. Since a considerable amount of separation of the water columns was anticipated under a certain condition, a total length of 12.40 meters of acryl resin pipe was used. As a result, the time needed for the pressure waves to propagate in the pipe is prolonged. The water passing through the mid-point of the pipe passes through the measurement holes $6,7,8$ and 9 before it flows into the reservoir. In order to main. tain the water in the reservoir at a constant level, the sectional area of the reservoir was made as large as possible (diameter, $1600 \mathrm{~mm}$ ) and a total of 8 outlets were installed on the wall of the reservoir at an interval of one meter. The water over flowing the reservoir returns through a 5 -in pipe to the original water pool. In order to prevent the changes in the water level occurring when the flow was reversed on account of the stoppage of the main pump, an auxiliary pump was installed. To raise the water level of the reservoir, compressed air was applied from an air source. Piping was arranged symmetrically with the mid-point of the pipe as its center, so that the effects of non-symmetry of the piping on the separation phenomenon could be reduc. ed as much as possible, and the radius of curvature was made large enough in order to prevent the reflection of the pressure waves generating from the bending parts. Generally, the longer the pipe, the easier the measuring. However, in the present experiment,

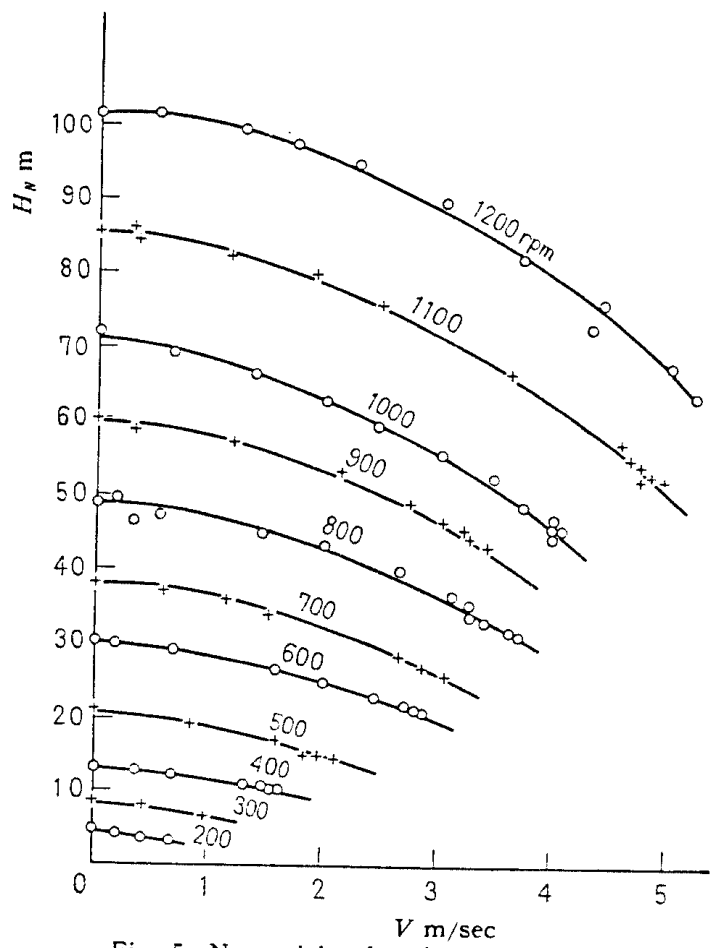

Fig. 5 Normal head and pump speed the total length of the pipe is 120.72 meters.

A. Main pump : $0.5 \mathrm{~m}^{3} / \mathrm{min}, 73.5 \mathrm{~m}, 1200 \mathrm{rpm}$, $12 \mathrm{~kW}$ Motor: $50 \mathrm{c} / \mathrm{sec}, 200 \mathrm{~V}, 9.7 \mathrm{kgm}, 120 \sim 1200$ $\mathrm{rpm}$

B. Electric valve: Dia. of the valve $53 \phi$, stroke of the valve $15 \mathrm{~mm}, 13$-speed changes chi)

C. Electro-magnetic flow meter: FMR-2 (Hita-

D. Semi-conductor pressure detector: PMS-5, $5 \mathrm{H}, 20 \mathrm{H}$ and $50 \mathrm{H}$ (Toyota Koki)

E. Pressure tank: Allowable pressure-3 atmospheric pressure, capacity : about $17 \mathrm{~m}^{3}$, weight : $3.5 \mathrm{t}$

F. Auxiliary pump: $0.26 \mathrm{~m}^{3} / \mathrm{min}, 30 \mathrm{~m}, 1450$ $\mathrm{rpm}, 3.7 \mathrm{~kW}$

G. Strain amplifier: CA 105 (Sanei Sokki)

H. \& I. DC Amplifier: DA 103 (Sanei Sokki)

J. Recorder : Electro-magnetic oscillator or handwriting oscillaor

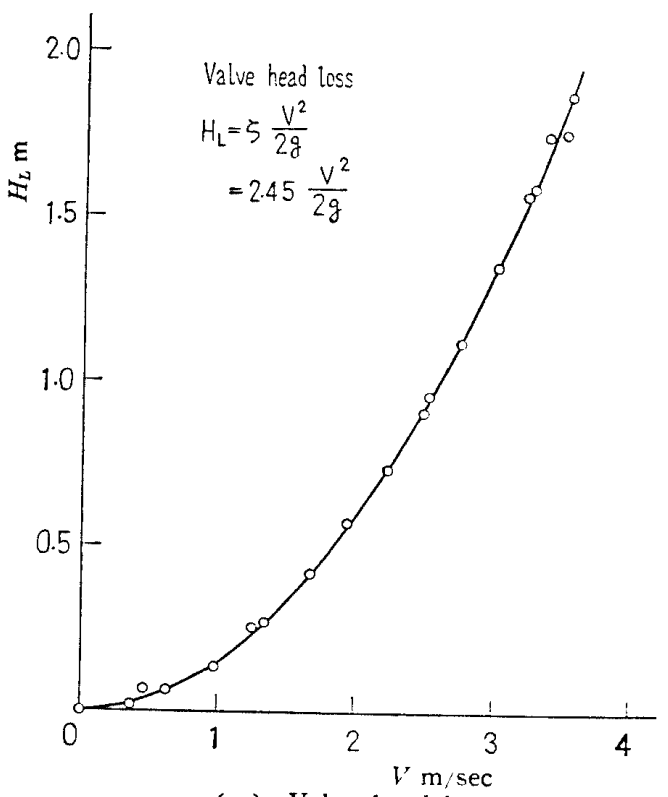

(a) Valve head loss

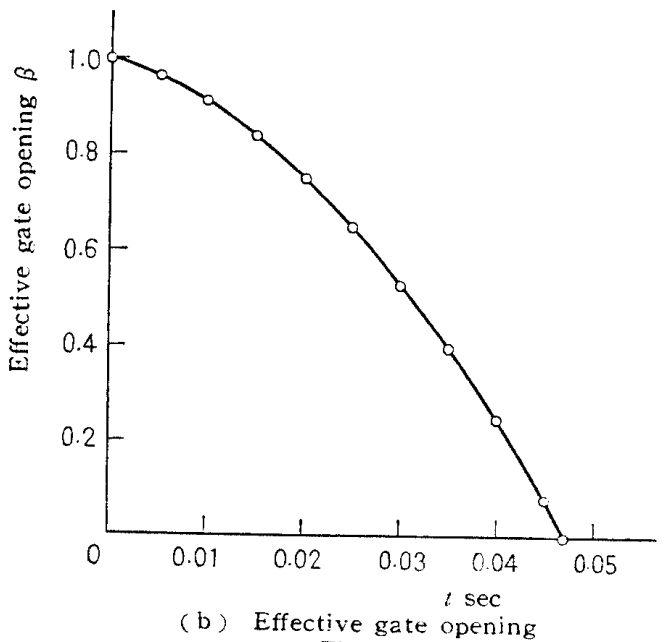

Fig. 6 


\section{Characteristics of the test apparatuses}

$5 \cdot 1$ Characteristics of the pump

The relationships between the number of revolutions of the pump and the normal head are shown in Fig. 5.

5.2 Characteristics of the valve

The head loss of the valve is shown in Fig. 6 (a), and an example of the time needed for closing and opening the valve is shown in Fig. 6 (b).

\subsection{Characteristics of the pipe}

The head loss of the pipe and its distribution are shown in Fig. 7 (a) and (b) respectively. These show that while the pipe bend causes practically no head loss, the acryl resin pipe in the central part causes a considerable form resistance. Also, the initial conditions at various points of the pipe are determined on the basis of the distribution curve.

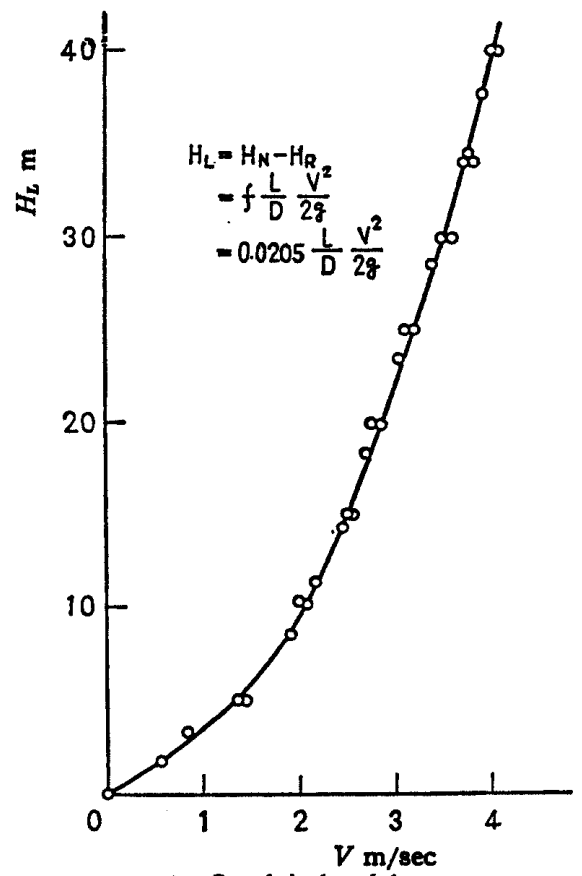

(a) Conduit head loss

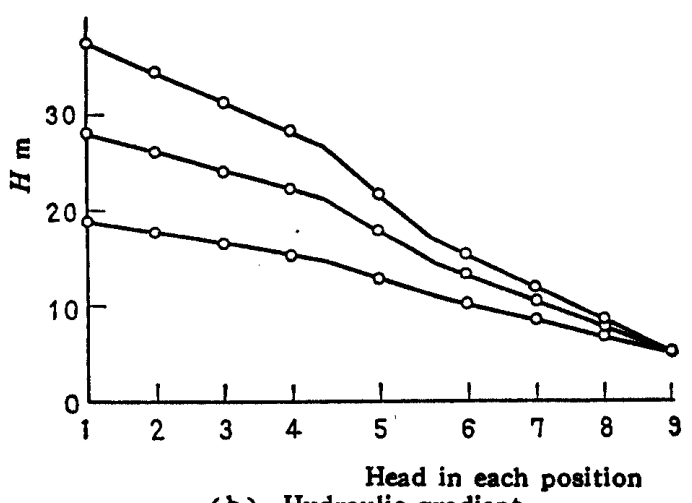

(b) Hydraulic gradient

Fig. 7

\section{Results and discussions}

6.1 Generation and disappearance of the water column separation

The separation of water columns generates a large number of air bubbles [Fig. 8 (a)]. A great majority of the air bubbles are generated in an area

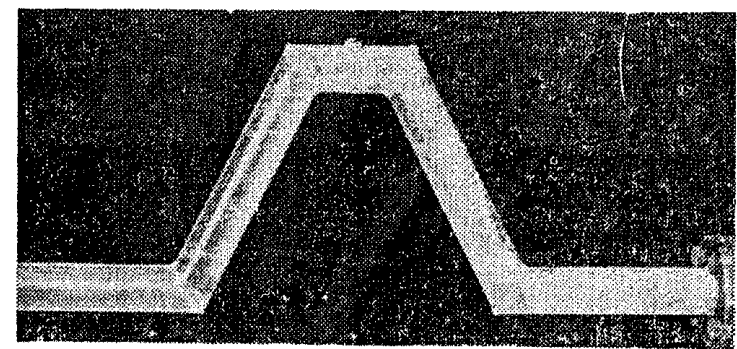

(a) Out-break of bubbles

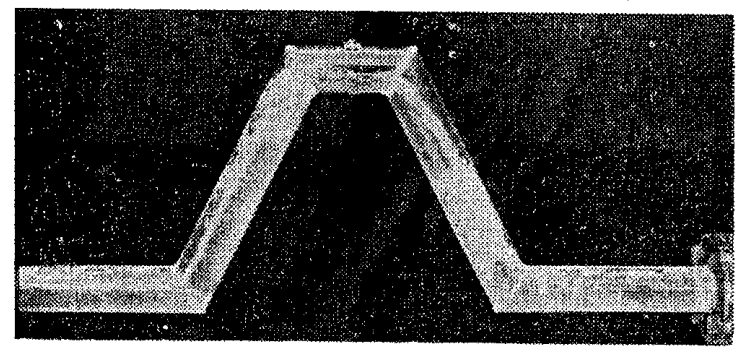

(b) Growth of cavities

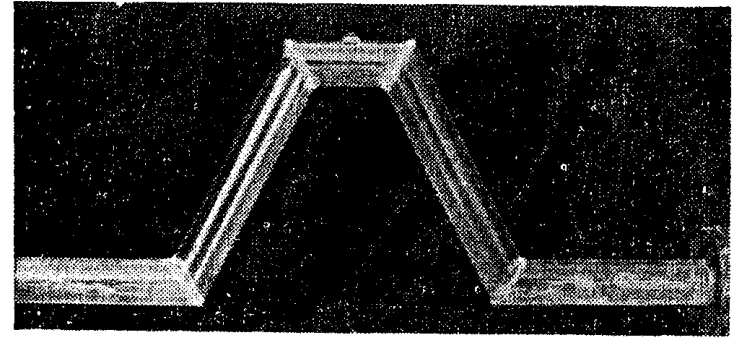

(c) Complete separation of water

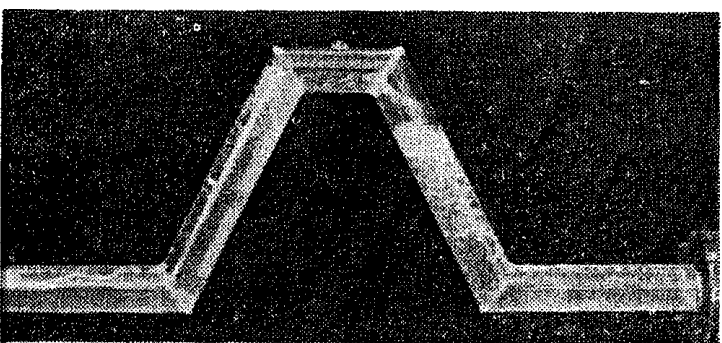

(d) Decay of vacuum

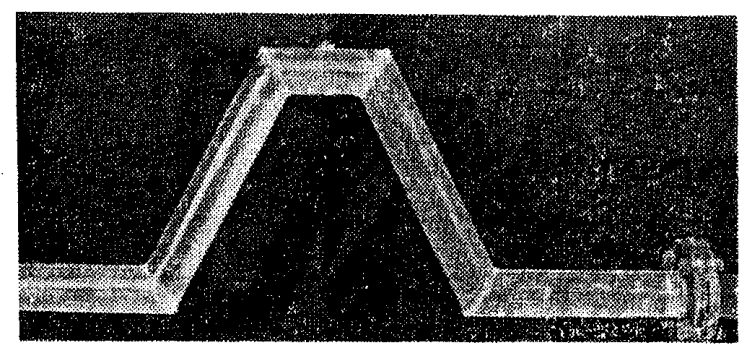

(e) Rejoining

Fig. 8 Formation process and collapse process 
ranging from the crest of the water column to the down stream, while a few of them are observable in the up stream. This indicates that the pressure in the down stream is lower than that in the up stream. It is, therefore, desirable to install vacuum breaker valves in the upper part of the down stream side in the condenser cooling water circulating sys. tem. The growing air bubbles create cavities in the pipe [Fig. 8 (c)]. Air bubbles are mixed with the cavities in the boundary adjoining the water column, thus making a complicated boundary. In the theoretical treatment of this phenomena, the boundary was considered as a part of free surface of water in calculation, but this treatment is problematical. Cavities are reduced in number at the moment the water starts to flow in reverse [Fig. 8 (d)] and finally, disappear when the water columns recombine themselves with a resounding noise in the pipe [Fig. 8(e)]. At such a moment, vibrations of considerable magnitude develop in the pipe and at the same time an extraordinary rise in the pressure is observed.

\subsection{Pressure rise and the time needed for} closing the valve

With a view to determining the relationships between the time needed for closing the valve and the initial pressure rise $H_{\max 1}$ and the second pressure rise $H_{\max 2}$, an experiment was conducted with a normal head $H_{N}=30 \mathrm{~m}$, and the water level of the reservoir $H_{R}=5 \mathrm{~m}$. The things to be noted in Fig. 9 (b) are the facts that the pressure rise

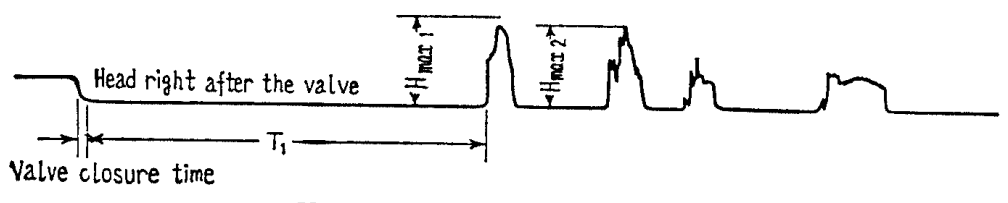

(a) Valve closure and pressure head rise

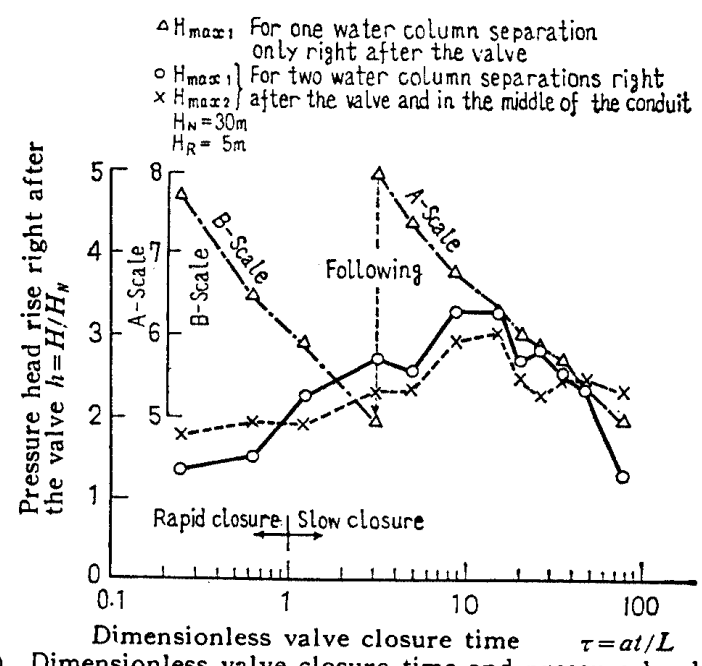

(b) Dimensionless valve closure time and pressure head rise does not necessarily correspond to the speedy closing of the valve and that $H_{\max 1}$ is not necessarily higher than $H_{\mathrm{max} 2}$. (Ordinarily, $H_{\mathrm{max} 1}$ is higher than $H_{\text {max2 }}$ ). Presumably, it is because of the fact that separation of water columns is taking place at two points : one at a point immediately after the valve and another at the central part. In other words, when the valve closes rapidly and causes large bubbles thereby, even if the water column developing in the up stream between the valve and the mid-point of the pipe hits the valve, there is not so much pressure rise because both ends are cushioned by the vapour pressure. The vapour in the center of the pipe acts to prevent the water column from hitting the valve. The weakness of inertia force is believed to have been caused by the division of the water column into two equal halves. It was shown that when the time needed for closing the valve is lengthened, the water column does not separate immediately after the valve and that the value of $H_{\mathrm{max}}$ declines rapidly. When this is compared with the results of an experiment in which the separation of the water column occurs only in the immediate rear of the valve (the height in the center is eliminated in this case), it becomes evident that the pressure rise resulting from an abrupt closure of the valve is extraordinarily high. Such a pressure rise is believed to have been caused by the combined force of the reflection pressure wave, originating from the reservoir, and the water column, generated in the absence of the high point in the center of the pipe.

6.3 Pressure rise and normal head

Experiments were conducted to measure the effects of the different normal heads on the pressure rise maintaining the time needed for closing the valve at a constant 0.047 seconds and $H_{R}$ at constant values on various levels $(0,5,10$, 15 and 20 meters), and the results are shown in Figs. 10 (a) and (b). Both $H_{\max 1}$ and $H_{\max 2}$ rapidly increased as $H_{N}$ was raised.

However, when $H_{R}$ is high, the pressure rise is not always high even under the same value of $H_{N}$. Thus, the phenomena are so complicated that one can only make an observation of the general trend. Also, in areas where $H_{N}$ was less than 20 meters, it was observed that $H_{\operatorname{maxi}}$ was lower than $H_{N}$ and this might be a remarkable characteristic attributable to the separation of the water column at two points. The dotted line indicates the results of the experiment without the high point in 
the center of the pipe, and it shows that, for $H_{R}=$ $5 \mathrm{~m}$, pressure rises are much larger than those of the case in which separation of water columns took place at two points.

6.4 Pressure rise and normal velocity of flow

The effects of the normal velocity of flow on the first pressure rise $H_{\max 1}$ are as shown in Fig. 11. According to the rigid theory, $H_{\mathrm{max}}=\left(a V_{N / q}\right) /$

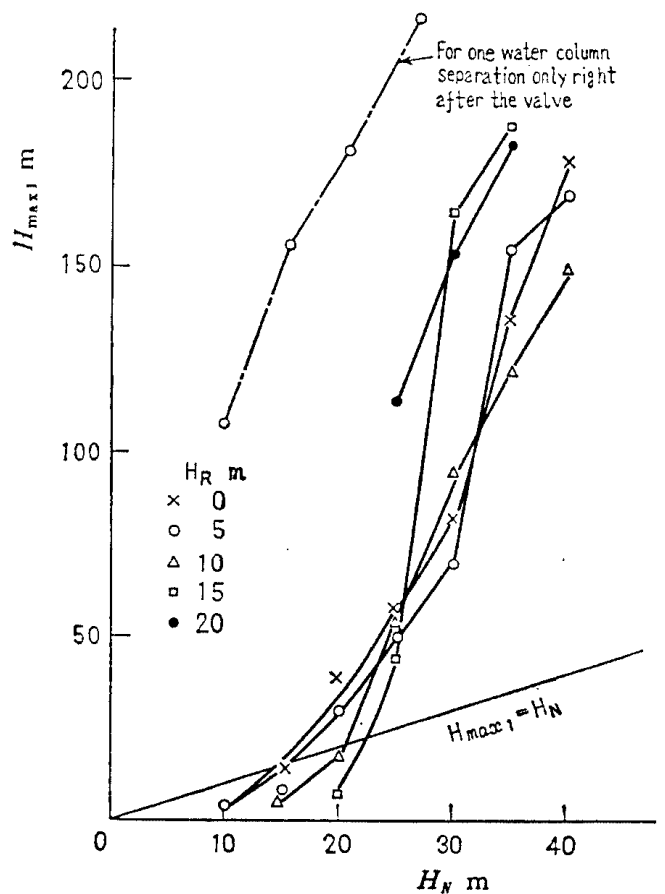

(a) Normal head and first pressure head rise

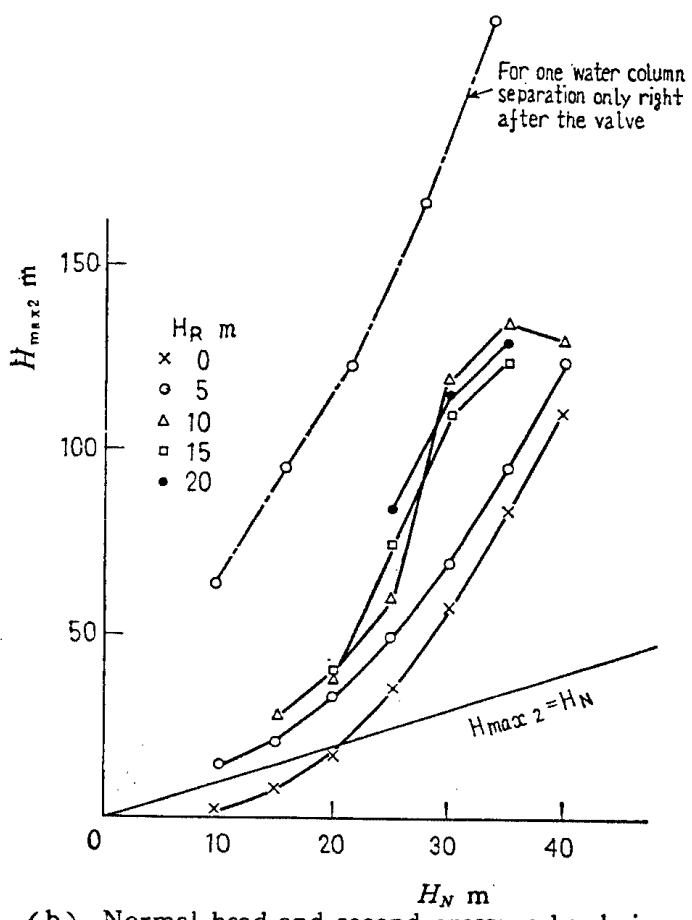

(b) Normal head and second pressure head rise Fig. 10 $\sqrt{1+f V_{N}^{2} / D B}$ is derived from Eq. (21), and since the second term of the denominator is small, it is proportional to the normal velocity of flow. However, this is not applicable to the results of the experiment and the pressure rise estimated by the rigid theory is exaggerated. In the case of the normal velocity of flow, the pressure rise becomes high as the value of $H_{R}$ is increased. Figures 10 (a), (b) and 11 indicate a peculiar phenomenon showing a rapid rise in the pressure under $H_{R}=20 \mathrm{~m}$, even if $H_{N}$ and $V_{N}$ are small, and this is caused by the fact, as it will be discussed later in this paper, that the water columns developing in the up and down streams combine themselves into one and hit the valve.

6.5 Pressure rise and reservoir's water level

Experiments were conducted to measure the effects of the water level of the reservoir on the pressure rise, maintaining $H_{N}=30 \mathrm{~m}$ and the time needed for closing the valve at a constant $0.26 \mathrm{sec}$ onds, and the results are shown in Fig. 12 (a). Initially, $H_{\max 1}$ is larger than $H_{\max 2}$, but when the water level of the reservoir $H_{R}$ rises above 7 meters, $H_{\max 2}$ becomes larger than $H_{\max 1}$. This is due to the fact that when the water level of the reservoir is raised, the volume of separation of the water column in the center of the pipe decreases and recombination occurs rapidly. The fact that $H_{\max 1}$ declines is attributable to the diminishing difference in the water level between the normal head, maintained constant and the reservoir level, as the latter is raised, and to the reduction of the

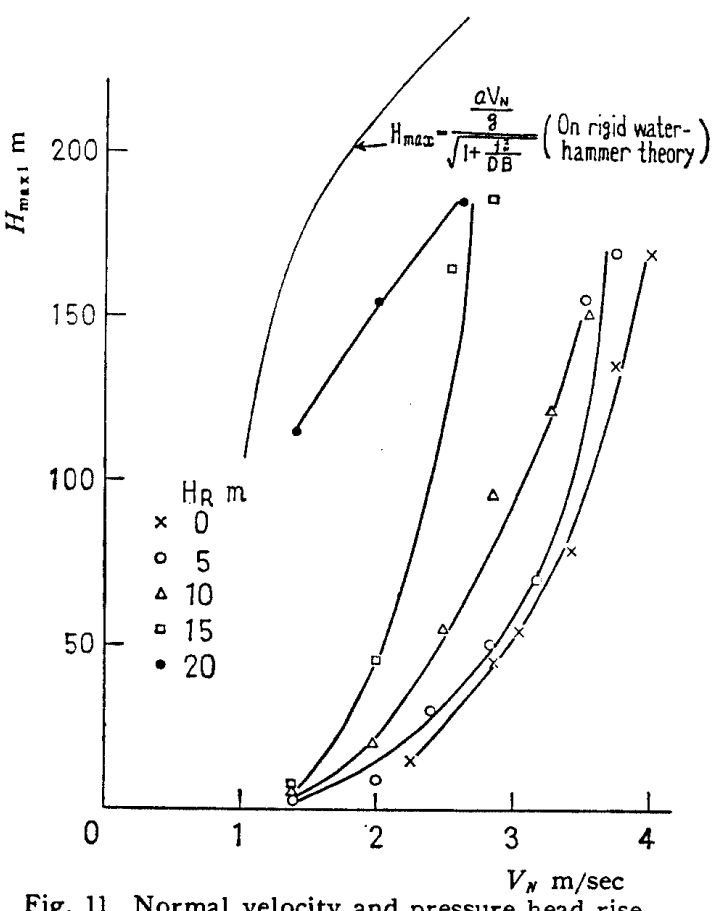

Fig. 11 Normal velocity and pressure head rise 
velocity of flow in the pipe i.e., the inertia force. In Fig. 12 (b) are shown the rejoining times of the water columns in the center of the pipe. From this it is apparent that if $H_{R}$ is increased, the rejoining is very much quickened.

6.6 Maximum pressure rise and pipe constant

In the fundamental equations based on the elastic theory, there are the four dimensionless values of $\rho^{*}, \zeta^{*}, \theta^{*}$ and $M^{*}$ which control the water hammer phenomena. If these dimensionless values and the boundary conditions are the same, a law of similarity of water hammer should hold. According to the classic theory, the terms of viscosity and inertia of these four dimensionless values are

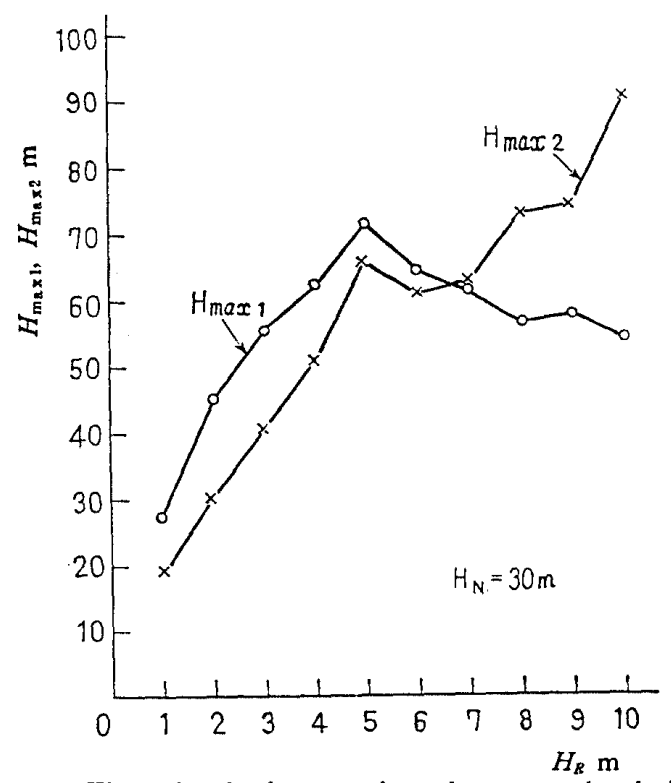

(a) Water level of reservoir and pressure head rise

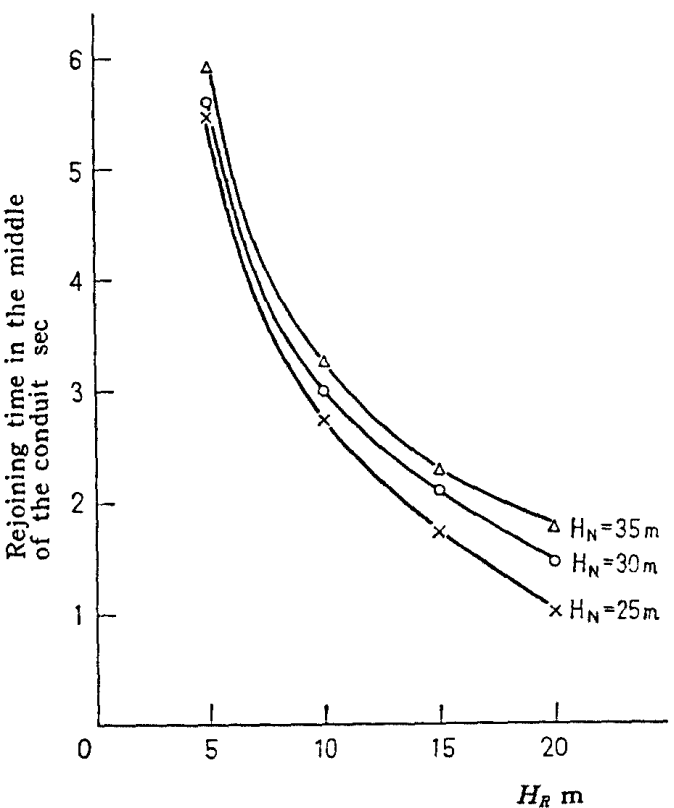

(b) Water level of reservoir and time of rejoining Fig. 12 dispensed with, and Eqs. (5) and (6) become $d h=\mp \rho^{*} d v$. Equation (22) in the rigid theory is

$$
h_{\max }=\frac{\rho^{*}}{\sqrt{1+\frac{\zeta^{*}}{h_{a}+h_{R}-h_{v}}}}
$$

and it is known that the pressure rise is proportional to the pipe constant $\rho^{*}$. However, the situation in Fig. 13 is complicated, when the separation of the water columns occurs both in the immediate rear of the valve and at the high point of the pipe. And in the case of the present experiment, it is. difficult to evaluate the pressure rise by a simple theory. However, if a refined theoretical calculation is made, taking the separation of the water columns into consideration, it is possible to make a close approximation of the pressure rise.

\subsection{Pressure rise at various points of pipe}

The pressure rise at various points of measurement on the pipe is shown in Fig. 14. Conditions: of the experiment are that the time needed for closing the valve is 0.52 seconds and the normal

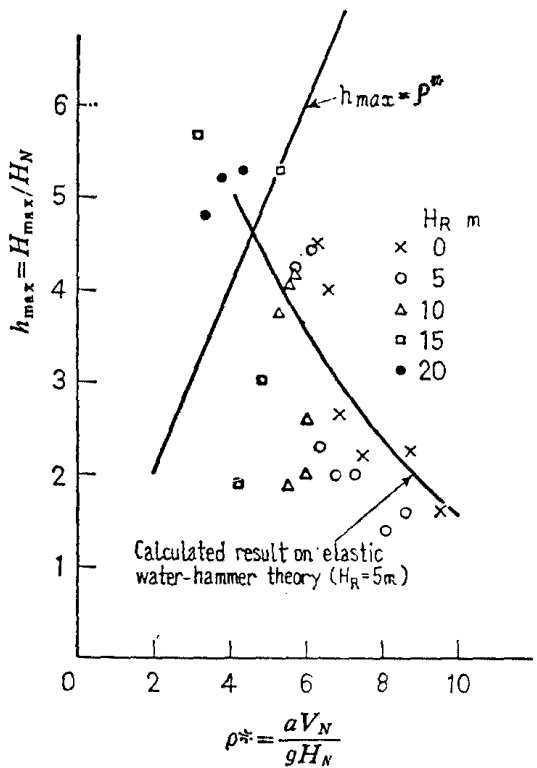

Fig. 13 Pipe constant and pressure head rise

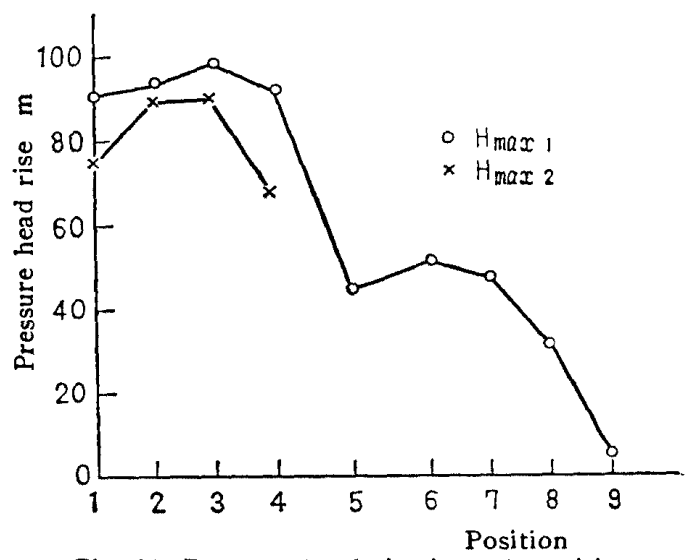

Fig. 14 Pressure head rise in each position 
velocity of flow is $V_{N}=3.2 \mathrm{~m} / \mathrm{sec}$. According to this figure, there are some big pressure rises in the up stream from the mid-point of the pipe, while there are not so large pressure rises in the down stream. This would indicate that in designing the pipe arrangement, care must be taken to strengthen the pipe on the side of the up stream.

6.8 Time of the pressure rise and normal velocity of flow

In the rigid theory, the time needed for the pressure rise $T_{1}$ is expressed by Eq. (28).

When $x=V_{N} \sqrt{\frac{f}{B \bar{D}} \text {, }}$

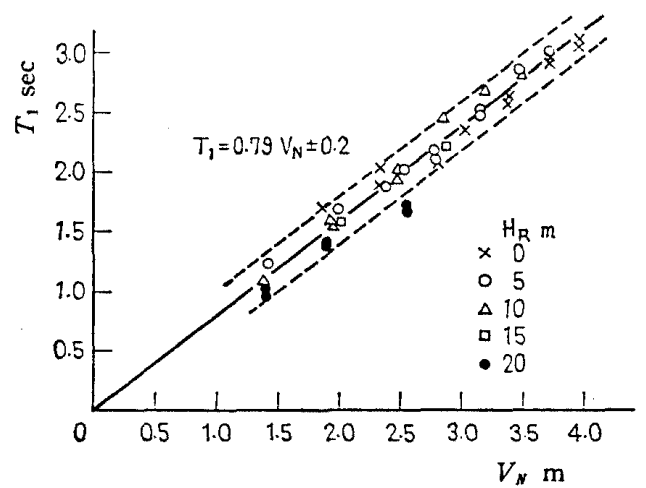

Fig. 15 Normal velocity and pressure-head-rise time by means of series expansion:

$$
\begin{aligned}
& \tan ^{-1} x=x-\frac{1}{3} x^{3}+\frac{1}{5} x^{5}-\cdots \cdots \\
& \log \frac{1+x}{1-x}=2\left(x+\frac{1}{3} x^{3}+\frac{1}{5} x^{5}+\cdots \cdots\right)
\end{aligned}
$$

we have

$$
T_{1}=\sqrt{\frac{D}{f B}}\left(4 x-x^{3}+\frac{11}{20} x^{5}-\cdots \cdots\right)
$$

Since $x$ is less than 1 , if only the first term is considered, it is expressed by

$$
T_{1}=\frac{4}{B} V_{N}+\varepsilon=\frac{2 L}{\left(H_{a}+H_{R}-H_{v}\right) g} V_{N}+\varepsilon
$$

Generally, the coefficient of $V_{N}$ is a function of $H_{R}$. Accordingly, if $H_{R}=0 \sim 20$ used in this experiment is applied, the coefficients become $1.2 \sim 0.4$. When $H_{R}$ is 5 meters,

$$
T_{1}=0.82 V_{N}+\varepsilon
$$

And an empirical formula applicable to the results of the experiments on the basis of Fig. 15 is expressed by

$$
T_{1}=0.79 V_{N} \pm 0.2
$$

which satisfies almost all requirements. In this case, the coefficient of $V_{N}$ is not a function of $H_{R}$, because the water column is broken into two parts at the high point of the pipe.

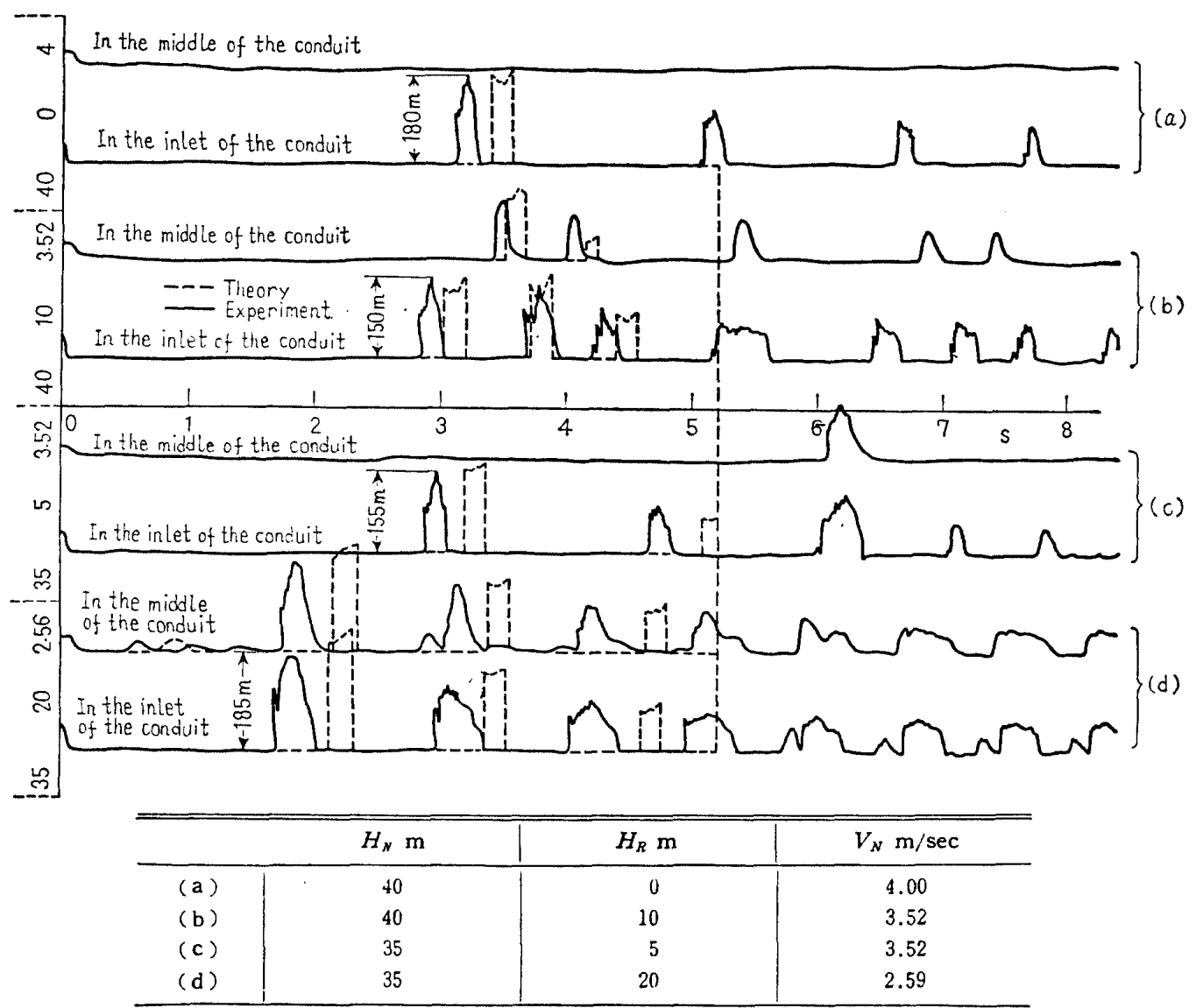

Fig. 16 Comparisons between experimental and calculated results on elastic water-hammer theory 


\subsection{Comparisons between the theories and the} experiments

In Fig. 16 are shown the results of comparison between the results of the theoretical calculation and those of the experiments. The upper part indicates the changes in the pressure occurring at the center of the pipe, while the lower part shows those occurring in the immediate rear of the valve. Generally, the theoretical values tend to put the pressure rise high and the time of recombination somewhat late. This is due to the fact that, in theoretical calculations, the boundary of the separation of the water columns is clearly identified as the free surface of the water. In Fig. 16 (a), the water in the latter half of the pipe is discharged out; any recombination of the water columns does not take place at the center. But some recombinations occur in the immediate rear of the valve, thereby they push the pressure rapidly upward. In Figs. 16 (b) and $(c)$, the water columns recombine in the immediate rear of the valve and next at the center of the pipe. In the case of Fig. 16 (c), due to the low water level of the reservoir, a large separation of the water columns takes place at the center. And the third pressure rise occurring in the imme. diate rear of the valve, which receives the impact of the recombination taking place at the center, is considerably large. The pressure rise at the center is quickened when the water level of the reservoir is raised.

In Fig. 16 (d) is shown that the first recombination of the water columns due to a raised water level of the reservoir occurs at the center of the pipe, and the second recombination of the water columns which hits the valve increases the pressure markedly. In this case, it is apparent that the velocity of the water column in the down stream is slightly faster than that in the up stream, that the pressure rise generated by the recombination of the water columns at the center is negligibly small, and that the effects of the refrection wave on the pressure rise in the immediate rear of the valve is much larger. The periodic times of wave gradually diminish due to the effects of the non-linear terms. The experimental values are more pronounced.

Judging from the above results as a whole, from the first up to the second pressure rises, the thcoretical calculations generally correspond to the experimental values, but they are no longer reliable with regard to the third pressure rise. However, what really counts in the designing of pipe arrange. ment is the large pressure rises at the first and the second instances. Therefore, as far as the discussions of these values are concerned, we believe the theoretical calculations will serve a useful purpose.

\section{$6 \cdot 10$ Surging phenomenon}

In the present experiment, a surging phenomenon of the following two conditions emerged. (a) $H_{N}=35, H_{R}=10$ : The time needed for closing the valve, 0.072 seconds. Figure 17 gives the record of this experiment. (b) $H_{N}=40, H_{R}=10$ : Time needed for closing the valve, 0.072 seconds. This phenomenon is related to the characteristic-frequency of the entire piping system. And it has been confirmed that this phenomenon is prone to occur when the bubbles in the immediate rear of the valve and those in the center simultaneously disappear.

\section{Conclusions}

(1) The theoretical calculation on the assumption that the boundary between the vapour part and a water column is the same as the free surface of the water still leaves something problematical. However, as far as this assumption is applied to the calculations of the first and the second pressure rises, the theory and the experiment accord pretty closely with each other, and this will facilitate practical application from the standpoint of engineering.

(2) For the condenser cooling water circulation system of thermal power plants, it is desirable to install vacuum breaker valves on the upper part of the down stream following the condenser. And the pipe on the up stream side should be stronger than that on the down stream side.

(3) When the separation of the water columns takes place at two points, the first pressure rise is not necessarily larger than the second pressure rise is not necessarily larger than the second pressure rise. The separation of the water column in the immediate rear of the valve acts to hamper the rise of pressure. Particularly when the water level of the reservoir is high and when the velocity of the reverse flow of the water column on the side of the down stream is great, it combines with the water column on the side of the up stream to increase the pressure markedly. Also, in such a case, surging phenomena are likely to occur in the piping system.

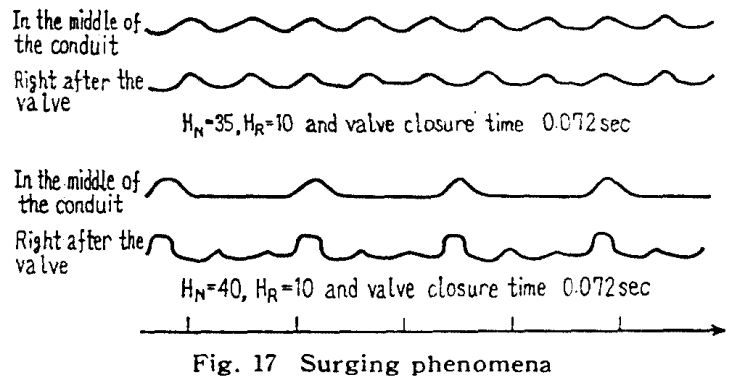




\section{Acknowledgement}

The authors wish to express their appreciation to Hideya Makino, Chikashi Tame and Novuo Okabe for their assistances in performing the experiments.

\section{References}

(1) J. Parmakian: Waterhammer Analysis, (1955), p. 86, Prentice-Hall.

(2) J. Parmakian: Trans. ASME, Vol. 80, No. 7 (1958), p. 1563.

(3) L. Bergeron: Waterhammer in Hydraulics and Wave Surges in Electricity, (1961), p. 79, John Wiley.

(4) R. W. Angus: Proc. Inst. Mech. Engrs, No. 136 (1937), p. 245.

(5) R. T. Richards: Trans. ASME, Vol. 78 (1956-8), p. 1297.
(5) J. Duc: Int. Symp. on Waterhammer in Pumped Storage Projects, ASME, (1965), p. 154.

(7) J. T. Kephart and K. Dauvis: Trans. ASME, Ser. D, Vol. 83, No. 3 (1961-9), p. 456.

(8) W. H. Li: Proc. Amer. Soc. Civ. Engrs., EM4 (1962-9), p. 97.

(9) W. H. Li: Proc. Amer. Soc. Civ. Engrs., EM6 (1964-12), p. 133.

(10) M. R. Carstens and T. W. Hagler : Proc. Amer. Soc. Civ. Engrs., HY6 (1964-11), p. 161.

(11) R. A. Baltzer: Trans. ASME, Ser. D, Vol. 89, No. 4 (1967-12), p. 837 .

(12) V. L. Streeter and C. Lai: Proc. Amer. Soc. Civ. Engrs., HY3 (1962), p. 79.

(13) T. Tanahashi and E. Kasahara: Bulletin of JSME, Vol. 12, No. 50 (1969), p. 206.

(14) T. Tanahashi and E. Kasahara: Bulletin of JSME, Vol. 12, No. 54, (1969), p. 1380. 